No simple correlation between bowel habit and age was observed.

There was no correlation between bowel habit and nationality.

Four per cent. of the industrial population and $16 \%$ of those seen in general practice regarded themselves as "constipated." The use of this term sometimes correlated with infrequent bowel actions, and sometimes with hardness of the stool, but often it bore no relation to bowel frequency or stool consistency.

A correlation was observed between increasing bowel frequency and the subjects' opinion of the stool as "loose."

Of the total population $19.7 \%$ (16\% in the industrial community, $29 \%$ in general practice) took laxatives. The difference between the two series with regard to laxative-taking can be ascribed to different age distributions within the samples. The proportion of subjects who took laxatives increased with age in both series. No subject under 20 took laxatives more than once weekly, and only three of the 266 subjects in this age group took any laxatives. Only $20 \%$ of those who took laxatives in the combined series considered themselves "constipated."

We thank Lord Trenchard, chairman of T. Wall and Sons (Meat and Handy Foods) Ltd., for agreeing to the undertaking of this survey in the three factories, and the staff of the Company Medical Service who made it possible. We thank Dr. H. N. Levitt for his help and encouragement with the survey in general medical practice.

REFERENCES

Hardy, T. L. (1945). Lancet, 1, 519.

Keele, C. A., and Neil, E. (1961). Samson Wright's Applied Physiology, 10th ed., p. 392. Oxford University Press, London.

Parks, J. W. (1943). M.D. Thesis, Cambridge.

Reid, J. J. A. (1956). Brit. med. f., 2, 25.

\title{
Cytomegalovirus as a Possible Cause of a Disease Resembling Infectious Mononucleosis
}

\author{
E. KLEMOLA,* M.D. ; L. KÄÄRIÄINEN,* M.D.
}

Brit. med. F., 1965, 2, 1099-1102

So long as the aetiology of infectious mononucleosis is unknown, diagnosis will depend on the criteria set. There is a conflict of opinion on whether the presence of heterophilic antibodies against sheep erythrocytes in an abnormally high titre should be regarded as a criterion for diagnosis. The majority of clinicians to-day employ the diagnosis infectious mononucleosis even when the heterophil agglutination or the Paul-Bunnell test is negative, provided the clinical picture of the disease is compatible with infectious mononucleosis. However, many authors are of the opinion that the aetiology of Paul-Bunnellpositive and that of Paul-Bunnell-negative mononucleosis are different (Shubert et al., 1954 ; Hoagland, 1955 ; Hobson et al., 1958 ; Belfrage, 1962).

A significant rise in the titre of complement-fixing (C.F.) antibodies to the cytomegalovirus was demonstrated in the course of the disease in the serum of a man admitted to the Aurora Hospital in February 1965. His illness was regarded on clinical grounds as infectious mononucleosis, though the heterophil agglutination test was negative. On the basis of the interesting rise observed, a systematic study on the behaviour of the C.F. antibodies to the cytomegalovirus was initiated in patients with infectious mononucleosis and-as a control-in patients with various acute infectious diseases.

\section{Material and Methods}

The material consists of a series of 14 patients with a clinical diagnosis of infectious mononucleosis admitted to the hospital in February, March, and April 1965, whose titre of C.F. antibodies to the cytomegalovirus has been followed for a minimum of six weeks from the onset of the disease. The haematological criteria for the diagnosis were relative and absolute lymphocytosis and an abundance, not merely a transient appearance, of atypical lymphocytes. Five patients had a negative and nine a positive heterophil agglutination test. The clinical features

\footnotetext{
* From Aurora Hospital and from the Department of Virology, Univer-
} sity of Helsinki, Helsinki, Finland. of the former are discussed below; all the latter had tonsillitis and enlargement of the lymph nodes. The limit for positivity was set at a titre of 1:32 after guinea-pig-kidney absorption ad modum Dawidsohn. In addition, the C.F. test was performed on paired serum samples taken during the disease and stored at $-20^{\circ} \mathrm{C}$. from 10 patients who had been in the hospital in 1964 for Paul-Bunnell-positive mononucleosis. Antibodies were studied for an average of eight months after the onset of the disease in two of these cases found to have a high titre.

The control material consists of 308 patients of different ages with a history of various acute infectious diseases. From 130 of these patients at least two serum samples were taken at intervals of two to four weeks.

Cytomegalovirus, strain "AD 169," isolated by Rowe et al. (1956) from adenoid tissue, was kindly supplied in 1959 by Dr. W. P. Rowe, Bethesda, Md., to Dr. P. Halonen, at that time of this laboratory. The virus was passaged several times in human embryo fibroblasts. The identity of the passaged virus was established in 1962 by the co-operation of Dr. T. H. Weller, Harvard University, Boston ; Dr. Janet Hartley, Bethesda, Md. ; Dr. Gun Carlström, Department of Virus Research, Karolinska Institute, Stockholm; and Dr. P. Halonen, Department of Virology, University of Turku. The identification was made on the basis of typical cytopathic changes caused by the virus and of C.F. tests (Carlström, 1965).

In this study the cytomegalovirus was cultivated in human embryo skin muscle cells derived from embryos 8 to 16 weeks old. The maintenance medium was $92 \%$ Eagle's minimum essential medium, $3 \%$ horse serum, and $5 \%$ tryptose phosphate broth. Antigen for the C.F. test was harvested when the complete cytopathic effect had appeared. The culture was frozen and thawed three times successively, and the fluid was either centrifuged to remove the cell debris or used without prior centrifugation. The C.F. test was performed by a microtechnique with Microtiter equipment (Cooke Engineering Co., Alexandria, Virginia). Antigen was first titrated with known positive convalescent human serum obtained from Dr. 
P. Halonen in a "box titration." In serum titrations all sera from the patient were tested simultaneously, using 4 antigen units and 1.5 to 2 units of complement. Virus serum dilutions and complement were incubated overnight at $4^{\circ} \mathrm{C}$. and for 15 minutes at room temperature before haemolytic system was added. Plates were then incubated for 60 minutes at $37^{\circ} \mathrm{C}$. and the result was recorded. Cups showing $25 \%$ haemolysis or less were regarded as positive and the serum titres were expressed as the reciprocal of the last positive serum dilution.

Virus-isolation experiments for cytomegalovirus were made on two patients from throat washings, sternal punctate, urine sediment, whole blood, and faeces by inoculating human embryo fibroblast cell tubes maintained in minimum essential medium supported with either $2 \%$ calf serum or $3 \%$ horse serum. The tubes were incubated at $35^{\circ} \mathrm{C}$. and the medium was changed one day after inoculation and every five days thereafter. The cell-culture tubes were examined daily. If no cytopathic changes were seen after four weeks the incubation isolation experiment was regarded as negative.

\section{Results of Virological Studies}

A significant rise in the titre of C.F. antibodies to the cytomegalovirus was demonstrated in all five mononucleosis patients without a positive heterophil agglutination test (Fig. 1).

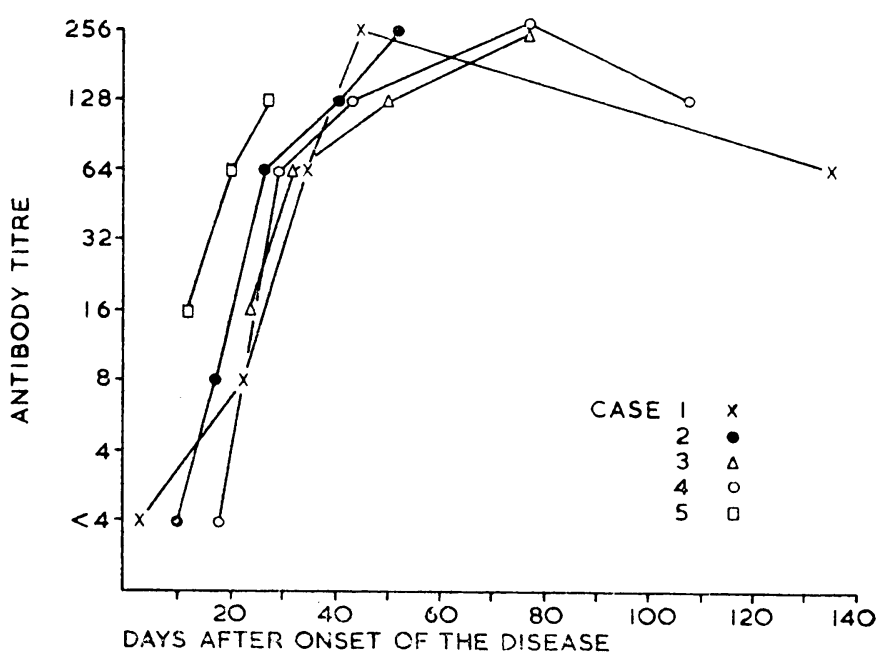

FIG. 1.-Course of development of C.F. antibodies to cytomegalovirus during the disease in patients with infectious mononucleosis without a positive heterophil agglutination test.

The titre values rose to 256 in three cases whose first titre was $<4$. The rise proceeded rather slowly and the maximum values were not established until more than six weeks from the onset of the disease. Our attempts to isolate cytomegalovirus have given negative results.

None of the 19 patients with Paul-Bunnell-positive infectious mononucleosis showed a significant rise of the titre of C.F. antibodies to the cytomegalovirus in the course of the disease. In nine cases no antibodies were detectable. A high titre (128) was demonstrable in two cases already in the first serum sample taken 8 to 14 days from the onset of the disease. An eightfold decrease in the titre was demonstrated in both cases later, after the disease was over.

A significant rise of the C.F. antibodies to the cytomegalovirus was not demonstrable in a single case of the control material. A high titre (128) was found in two patients, both of whom had a chronic debilitating disease in addition to pneumonia.

${ }^{1}$ This serum has been used as control positive serum since 1960 , and it has been titrated with several lots of cytomegalovirus C.F. antigens.
The distribution of the titre values of the controls can be seen in Fig. 2.

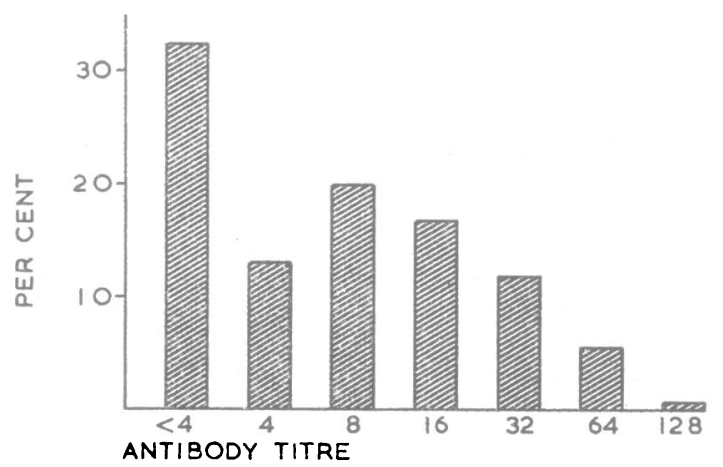

FIG. 2.- Percentage distribution of titres of C.F. antibody to cytomegalovirus for the 308 patients of the control material.

\section{Clinical Features in Patients with a Rise in C.F. Antibodies to Cytomegalovirus}

For the five patients with a significant rise in the titre to the cytomegalovirus in the course of the disease the clinical diagnosis was, as mentioned above, infectious mononucleosis without a positive heterophil agglutination test. The titre of heterophil agglutinins after the guinea-pig absorption test was continuously less than $1: 4$ in four cases. The patients were adult men with the exception of a girl aged 1 year 10 months (Table I). The cases occurred in Helsinki in the course of

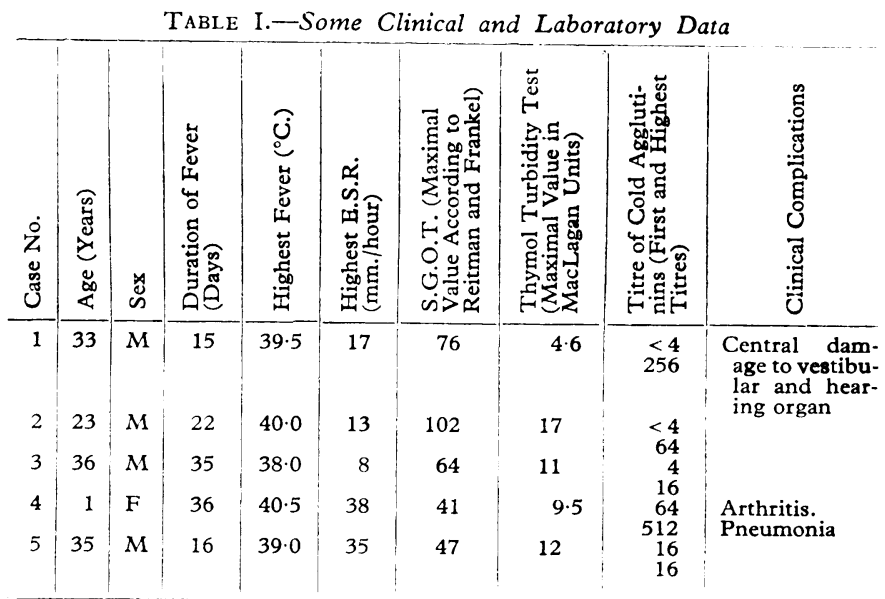

three months. The exceptionally high incidence of this kind of mononucleosis aroused attention at the hospital. However, no epidemiological connexion between the cases was noted. Pyrexia lasted for two to five weeks ; it was intermittently high in three cases. However, the general condition of all the patients except the child remained good. Tonsillitis was not present in a single case. Mild enlargement of cervical and axillary lymph nodes of short duration was established in only one patient (Case 2). All the adult patients complained of headache or pain in the back or limbs in at least some phase of the disease. Cerebrospinal fluid was normal in the two cases in which it was examined (Nos. 1 and 4). None of the patients had coryza, but all but one (Case 3 ) had cough. The results of the liver-function tests were abnormal in all cases (Table I), but the liver was distinctly enlarged in only one case (No. 4). The result of the thymol turbidity test was abnormal (14 MacLagan units) even three months after the disease in one case (No. 2). The serum bilirubin and alkaline phosphatase were normal in all the cases. The paper electrophoresis and immunoelectrophoresis patterns were normal in the two patients 
on whom these examinations were performed (Cases 3 and 4) about one month after the onset of the disease.

Case 1 was complicated by symptoms which, according to the otologist, were caused by central damage to the vestibular and hearing organ. Vertigo was severely disabling and persisted for four weeks. Occasionally nystagmus, tinnitus, and nausea occurred.

Case 4, a child whose mental and physical development had previously been perfectly normal, had joint symptoms for three weeks from the onset of the disease: one knee swelled first, and when it improved the other knee became swollen. The finger- and toe-joints were tender. There was a red skin rash with small spots for several days. Chest radiography revealed pneumonia; hilar shadows were accentuated and fine-fibred infiltrates were seen in the basal parts of the lungs. The E.C.G. was normal, as in all the other cases. The pneumonia and arthritis cleared up completely. The child appeared to be physically and mentally healthy at the follow-up two months after discharge from hospital.

The leucocyte count was low or normal $(4,000-6,500)$ and the number of mononuclear cells was less than $50 \%$ of the leucocytes during the first or second week for three cases studied in a relatively early stage of the disease (Nos. 1, 2, and 4). Changes characteristic of infectious mononucleosis were seen at the latest two weeks after onset, and they lasted for at least two to four weeks, after which they gradually subsided. Atypical lymphocytes, as described by Downey, accounted for over $1,400 / \mathrm{c} . \mathrm{mm}$. when the leucocyte count was highest in all the cases and for over $15 \%$ of total leucocytes with the exception of one case (Table II). In addition, a high proportion of the

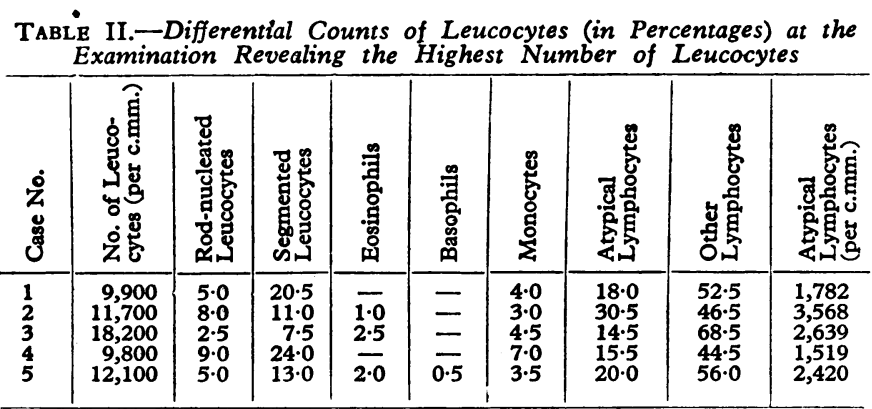

other lymphocytes were large and to some extent abnormal. One patient (Case 4) had slight anaemia. The thrombocyte count was normal. In the two cases studied the sternalpuncture finding was the same as that usually encountered in mononucleosis.

Blood culture was performed in two cases (Nos. 2 and 4) with negative results. The antistreptolysin and antistaphylolysin titres were within normal limits. Dye tests (Sabin-Feldman) and C.F. tests against toxoplasma were performed in all cases during the acute phase and six weeks after onset, with negative results. Serological tests for syphilis, salmonella, and leptospira infections and for Listeria monocytogenes were also negative. A significant rise of the cold agglutinin titre according to the method of Feller and Hilleman was observed in four of the cases during the disease (Table I). C.F. antibodies against Eaton pleuropneumonia-like organisms did not rise in a single case. C.F. antibodies to numerous viruses-in Case 1 to 20 possible aetiological agents-were studied in all the cases, but with negative results.

\section{Discussion}

Congenital cytomegalic inclusion disease (C.I.D.) is a serious disease of infants, and in all probability is the result of an infection acquired in utero from an asymptomatically infected mother. C.I.D. is extremely rarely found in adults. Apart from their own 14 cases, Wong and Warner (1962) found only 41 cases reported in the literature. In the adult the disseminated form commonly occurs as a terminal complication of a serious debilitating disease.
C.F. and neutralizing antibodies to the cytomegalovirus have been found in a high percentage of human sera (Rowe et al., 1956 ; Weller et al., 1957). The older the age group the higher is the percentage. C.F. antibodies to the cytomegalovirus (at a titre of $\geqslant 4$ ) were established in a population study (Halonen and Kääriäinen, to be published) of 626 persons in Finland. They were encountered in $47 \%$ in the age group $10-12$ years, in $68 \%$ in the age group $15-35$ years, and in $81 \%$ in the age group 36-60 years. The results were thus similar to those obtained elsewhere (Rowe et al., 1956 ; Carlström, 1965). Hence it seems that post-natally acquired cytomegalovirus infections are common and usually not apparent. The observation that cytomegaloviruses isolated from man are not antigenically homogeneous (Weller et al., 1960; Weller and Hanshaw, 1962) is very important, since it is possible that various members of this virus group differ in pathogenicity. C.I.D. has been the only disease in man attributed to the cytomegalovirus group, but on the basis of a recent study (Hanshaw et al., 1965) it seems that an acquired cytomegalovirus infection may sometimes play a part in the aetiology of unexplained hepatomegaly or chronic liver disease in children. The observation that C.F. antibodies to the cytomegalovirus rise in some patients with a clinical diagnosis of infectious mononucleosis without a positive heterophil agglutination test is, to the best of our knowledge, new. The validity of our findings is supported by the fact that a fourfold rise in the titre of C.F. antibodies to the cytomegalovirus was not found in a single case of our control material with various acute infectious diseases. The occurrence of the antibodies in this material was similar to that in a healthy population studied earlier (Halonen and Kääriäinen, to be published).

A rise in the titre of the antibodies to the cytomegalovirus may be due to the reactivation of a latent cytomegalovirus infection in the course of a disease caused by some other infectious agent. It would be strange for a phenomenon of this type to occur regularly in connexion with some special and not very serious infection. The observation that no antibodies to the cytomegalovirus were demonstrable in the first serum samples of three patients with a subsequent rise of the titre makes it more likely that the same agent-on the basis of similar antigenic properties a virus of the cytomegalovirus group -causes both the serological reaction to the cytomegalovirus and the disease itself. It is probable, however, that the syndrome infectious mononucleosis may, in the absence of a positive heterophil test, be caused by many different aetiological agents. Siim (1960) has found that acquired toxoplasmosis can cause haematological changes resembling those in infectious mononucleosis. We have observed previously at the Aurora Hospital, as Belfrage has in Sweden, the occurrence of infectious mononucleosis without a positive heterophil agglutination test and without tonsillitis, especially in the older age group. However, instead of sporadic cases there was a suggestion of an epidemic on this occasion.

As regards infectious mononucleosis with a positive heterophil agglutination test, an interesting observation, the significance of which it is impossible to assess so far, was made in our study in which all the patients of this group had the anginoseglandular type of the disease. A significant rise of the C.F. antibody titre to the cytomegalovirus was not observed in a single case. In two cases, however, a high titre was already demonstrable in the first serum sample, there being a significant decrease of the titre later, after the disease was over. The behaviour of the C.F. antibodies to the cytomegalovirus in these patients was quite different from that demonstrated in the patients without a positive Paul-Bunnell test. The subject requires further study.

\section{Summary}

A significant rise in the titre of complement-fixing (C.F.) antibodies to the cytomegalovirus strain " $\mathrm{AD} 169$ " was 
established during the disease in four previously healthy adults and one child with a mononucleosis-like disease without a positive heterophil agglutination test. No antibodies were demonstrable in the first sample from three patients.

The disease was characterized by protracted fever and absence of tonsillitis. Enlargement of the lymph nodes was seen in only one patient. The tests for liver function gave abnormal results in all the cases. Leucocytosis, relative and absolute lymphocytosis, and the appearance of abundant atypical lymphocytes were features of the haematological pattern.

No significant rise of the C.F. antibody titre to the cytomegalovirus was demonstrable in a single case among 19 patients suffering from infectious mononucleosis with a positive heterophil agglutination test or among 130 patients with various acute infectious diseases. A high titre, however, was found in two patients with infectious mononucleosis, there being a significant decrease of the titre later, after the disease was over, and in two patients of the control material, both of whom had a chronic debilitating disease in addition to their acute infection.

We are indebted to Dr. P. Halonen, now Professor of Virology, University of Turku, for making available the "AD 169 " strain of cytomegalovirus and for helpful advice, and to the following virologists and microbiologists for their advice and co-operation in the study of the aetiology: Professor N. Oker-Blom, Department of Virology, University of Helsinki ; Drs. O. Wager and E. Jansson, Microbiological Laboratory, Aurora Hospital ; and Dr. K. Cantell, State Serum Institute. We also express our gratitude to Mrs. P. Leino, Mrs. P. Koivunen, and Miss S. Keränen for technical assistance.
ADDENDUM.- In addition to the rise in the titre of complement-fixation antibodies, a significant rise in the titre of neutralizing antibodies to the cytomegalovirus has been established in all the five patients with a mononucleosis-like disease discussed above. Six further cases belonging to the category "infectious mononucleosis without a positive heterophil agglutination test" have been studied in the ensuing three months. In four of these-adults with the typhoidal type of the disease, without tonsillitis, and without enlargement of lymph nodes-a significant rise of complement-fixation antibodies to the cytomegalovirus has been demonstrated. On the other hand, no complement-fixation antibodies to the cytomegalovirus were demonstrable in the other two-children under 3 years of age, both of whom had exudative pharyngitis and enlarged lymph nodes.

\section{REFERENCES}

Belfrage, S. (1962). Acta med. scand., 171, 531.

Carlström, G. (1965)... Acta. paediat. scand., 54, 17.

Halonen, P., and Kääriäinen $L$ to be published.

Hanshaw, J. B., Betts, R. F., Simon, G., and Boynton, R. C. (1965) New Engl.' Ұ. Med., 272, 602.

Hoagland, R. J. (1955). Amer. F. med. Sci., 229, 262

Hobson, F. G., Lawson, B., and Wigfield, M. (1958). Brit. med. F., 1 845 .

Rowe, W. P., Hartley, J. W., Waterman, S., Turner, H. C., and Huebner, R. J. (1956). Proc. Soc. exp. Biol. (N.Y.), 92, 418.

Shubert, S., Collee, J. G., and Smith, B. J. (1954). Brit. med. F., 1, 671 Siim, J. (1960). In Human Toxoplasmosis, pp. 53-79. Wiliams \& Wilkins, Baitimore.

Weller, T. H., and Hanshaw, J. B. (1962). New Engl. 7. Med., 266, 1233.

- 1 and Scott, D. E. (1960). Virology, 12, 130.

- Macauley, J. C., Craig, J. M., and Wirth, P. (1957). Proc. suc. exp. Biol. N.Y.', 94, 4 .

Wong, T. W., and Warner, N. E. (1962). Arch. Path., 74, 40 s.

\title{
Hereditary Coproporphyria with Acute Intermittent Manifestations
}

\author{
H. D. BARNES,* M.SC., PH.D. ; NORMAN WHITTAKER, † M.A., M.D., M.R.C.P.
}

Brit. med. F., 1965, 2, 1102-1104

Detailed studies of cases of porphyria in recent years have shown the existence of two distinct forms of this disorder in which patients are liable to develop acute intermittent abdominal crises. Both forms are familial, and their patterns of inheritance indicate an autosomal dominant genetic basis. The first of these, widely known as acute intermittent porphyria, is prevalent in Sweden, where it has been intensively studied by Waldenström $(1937,1957)$ and Waldenström and Haeger-Aronsen (1963); the second form, for which the name variegate porphyria has been adopted, has its highest incidence in South Africa, where it has been studied and reported by Dean and Barnes (1955, 1959), Eales (1963), and by Eales et al. (1963). The principal differential features can be tabulated as follows :

\section{Acute Intermittent}

Intermittent episodes of acute abdominal pain, neurological disorder, and psychotic disturbance order, and psychouc disturbance in different proportions.

Urinary excretion of aminolaevulic

acid (A.L.A.) and porphobilinogen (P.B.G.)-porphyrin precursors - increased during attacks, with a marked tendency 10 rersist into remissions. Latent cases can be detected by appropriate tests.

stool porphyrins normal in the maiority of patients. The slight increases sometimes found can increases sometimes found can
probably be attributed to other causes.
The patients whose cases are reported below belong to one family, and the findings are not in strict accord with either of the above groups. The propositus suffered an acute porphyric episode towards the end of 1962 and on recovery his urine and stool were analysed in detail for porphyrins and precursors. Specimens were also obtained from all four of his sibs, and three of them showed the same anomalous pattern of excretion. The methods used in these analyses were those described by Rimington (1961) for porphyrins, and by Mauzerall and Granick (1956) for aminolaevulic acid and porphobilinogen.

\section{Case Report}

A man aged 22 was admitted to the North Middlesex Hospital on the surgical side under the care of $\mathrm{Mr}$. T. M. Hennebry on 1 November 1962, with a diagnosis of constipation and possibly appendicitis. He had had an attack of tonsillitis two to three weeks previously, and since then had been taking Soneryl sleepingtablets. His father, one brother, and one sister had been subject to abdominal pains, for which there was no definite diagnosis.

Clinical examination revealed an afebrile patient with a pulse of 110 and slight tenderness in the right iliac fossa. Hard faeces were felt in the rectum and there was tenderness on the right side of the pelvis ; his bowels had not opened for three days before admission. Five more days passed without evacuation of faeces;

\footnotetext{
* Medical Research Council Grantholder, St. Mary's Hospital Medical School, London.

† Consultant Physician, North Middlesex Hospital, London.
} 